Research Article

\title{
Path Planning and Control of a Quadrotor UAV Based on an Improved APF Using Parallel Search
}

\author{
Tianpeng Huang, ${ }^{1}$ Deqing Huang $\mathbb{D}^{1},{ }^{1} \mathrm{Na}$ Qin, ${ }^{1}$ and Yanan $\mathrm{Li}^{2}$ \\ ${ }^{1}$ School of Electrical Engineering, Southwest Jiaotong University, Chengdu, China \\ ${ }^{2}$ Department of Engineering and Design, University of Sussex, Brighton BN1 9RH, UK
}

Correspondence should be addressed to Deqing Huang; elehd@home.swjtu.edu.cn

Received 13 February 2021; Revised 2 June 2021; Accepted 22 June 2021; Published 15 July 2021

Academic Editor: Yue Wang

Copyright (c) 2021 Tianpeng Huang et al. This is an open access article distributed under the Creative Commons Attribution License, which permits unrestricted use, distribution, and reproduction in any medium, provided the original work is properly cited.

\begin{abstract}
Control and path planning are two essential and challenging issues in quadrotor unmanned aerial vehicle (UAV). In this paper, an approach for moving around the nearest obstacle is integrated into an artificial potential field (APF) to avoid the trap of local minimum of APF. The advantage of this approach is that it can help the UAV successfully escape from the local minimum without collision with any obstacles. Moreover, the UAV may encounter the problem of unreachable target when there are too many obstacles near its target. To address the problem, a parallel search algorithm is proposed, which requires UAV to simultaneously detect obstacles between current point and target point when it moves around the nearest obstacle to approach the target. Then, to achieve tracking of the planned path, the desired attitude states are calculated. Considering the external disturbance acting on the quadrotor, a nonlinear disturbance observer (NDO) is developed to guarantee observation error to exponentially converge to zero. Furthermore, a backstepping controller synthesized with the NDO is designed to eliminate tracking errors of attitude. Finally, comparative simulations are carried out to illustrate the effectiveness of the proposed path planning algorithm and controller.
\end{abstract}

\section{Introduction}

In recent years, UAV has been used in various applications, such as infrastructure management [1], logistics delivery [2], and estimation of aboveground biomass of mangrove ecosystems [3]. The implementation of UAV in all these applications requires it to follow a predefined path. In addition, to achieve path tracking, a good control system should be provided. Therefore, path planning with automatic obstacle avoidance and control are two essential tasks in UAV.

Research on UAV control has been extensively reported in the literature. In [4], a proportional-integral-derivative (PID) controller is designed to accomplish altitude and attitude tracking for a quadrotor helicopter. A fuzzy PID control method is proposed in [5], where the fuzzy rules are employed to automatically adjust the three parameters of PID controller. In [6, 7], an active disturbance rejection control (ADRC) scheme is developed to achieve trajectory tracking of a quadrotor UAV. PID and ADRC are model-free control strategies, which have an advantage of a simple control structure. However, it is difficult to tune the parameters of these two controllers.

In [8], an adaptive sliding mode control (SMC) is investigated to stabilize a quadrotor system subject to unknown external disturbance. [9] presents a continuous SMC approach to follow the predefined trajectories in position and attitude channels for a four-rotor UAV. An adaptive finite-time attitude tracking algorithm is applied to a quadrotor in the presence of uncertainty and disturbance in [10]. In [11], a disturbance observer is integrated to $H_{\infty}$ technique to 
realize hovering control of a quadrotor. The robustness of such a method has been verified by experiments. In [12], the differential flatness is used in tracking control of translational and rotational movements of an UAV system considering modeling uncertainty and disturbance. An adaptive linear quadratic control strategy is proposed in [13] to stabilize three attitude angles of a quadrotor. To achieve trajectory following of position and attitude subsystems, a nonlinear disturbance observer- (NDO-) based backstepping controller is proposed in [14], where the NDO is utilized to estimate external disturbance.

The bioinspired algorithms have been applied to path planning of UAV. In [15], a chaotic artificial bee colony (ABC) method is developed to design a path in complex environments. In [16], an ant colony optimization (ACO) algorithm is proposed to achieve trajectory planning for a UAV. [17] presents a particle swarm optimization (PSO) algorithm to address path planning of UAV. Genetic algorithm (GA), as a popular optimization algorithm, has been employed to plan a path in UAV [18]. Furthermore, a comparison of GA and PSO for real-time path planning of UAV is carried out in [19], where the results indicate that, under the consideration of statistical significance, the trajectories produced by GA are superior compared to that produced by PSO when using the same encoding. In $[20,21]$, a grey wolf optimizer is used to search a feasible and effective path for a UAV. An improved fruit fly optimization algorithm is introduced in [22] to address the problem of path planning of multiple UAVs in 3D complicated environments with online changing tasks. In [23], a flower pollination algorithm based on neighborhood global learning is employed to complete route planning of a UAV. [24] offers an evolutionary algorithm based on a novel separate evolution strategy to plan an optimized path for a single UAV. Furthermore, a constrained differential evolution is put forward to achieve path planning of a UAV in [25].

Besides the aforementioned bioinspired intelligent algorithms, there are many effective strategies to solve the problem of path planning of UAV. [26] studies a distributed multiagent path planning algorithm for quadrotors in dynamic environments. An energy-based path planning framework is used to improve flight endurance for a solarpowered UAV in [27]. A multiobjective path planning is presented in [28] to design a feasible path for a UAV, where safety is considered in the proposed algorithm. [29] introduces a path planning system based on an elliptical tangent model to reduce computational burden for a quadrotor UAV in an unknown unstructured outdoor environment. In [30], a ground feature-oriented approach is investigated to generate a suitable path for UAV mapping. Two path planning algorithms are designed in [31], one of which is based on the exact penalty method and successive convex approximation, and the other adopts a heuristic approach. In addition, [32] presents an improved A-star algorithm to generate a path for target tracking of a UAV. In [33], a Voronoi diagram-based multiple UAV path planning method is proposed to cooperatively attack multiple targets in a static environment. An improved rapidly exploring random tree (RRT) algorithm is introduced in [34] to realize 3D path planning of a UAV.
As an efficient path planning algorithm, APF has been applied to some scenarios, such as mobile robots [35, 36] and automated vehicles [37]. The traditional APF (TAPF) has two shortcomings, i.e., local minimum and unreachable goal. To address these problems, the repulsive potential function of TAPF is replaced by Gaussian function in [38]. However, UAV still might fall into a local minimum using the improved APF in [38] when obstacle is on the line connecting current position and target position. Moreover, when multiple obstacles are around target and repulsive gain is large, the resultant repulsive forces might be equal to attractive force, in which case UAV could not approach target. Motivated by the above analysis, a novel APF based on parallel search is proposed for path planning of UAV in this paper.

The main contributions of the paper are summarized as follows:

(1) A parallel search algorithm is proposed to address local minimum and unreachable target with obstacles nearby in TAPF

(2) Compared with existing results of path planning algorithms [34, 39, 40], a shorter path and less time consumption are obtained using the proposed algorithm

(3) Compared with ADRC [6, 7], better tracking performance is obtained by the proposed controller based on NDO with exponential convergence when following the planned path

The remainder of this paper is organized as follows. In Section 2, TAPF is introduced and the problems of local minimum and unreachable goal of TAPF are analyzed. In Section 3 , a novel APF based on parallel search is presented. Section 4 introduces the design of the observer and controller of the quadrotor. In Section 5, comparative simulations are conducted to verify the effectiveness of the proposed algorithm and controller. Section 6 concludes the work.

\section{TAPF Applied to Path Planning of UAV}

2.1. TAPF. APF is a virtual potential field in space, which consists of attractive potential field generated by target position and repulsive potential field generated by obstacle. The UAV automatically plans a path to destination under the influence of attractive potential field and repulsive potential field.

Let $\mathbf{P}_{\text {cur }}\left(x_{\text {cur }}, y_{\text {cur }}\right)$ and $\mathbf{P}_{\text {tar }}\left(x_{\text {tar }}, y_{\text {tar }}\right)$ represent the current position and target position of the UAV, respectively. Then, the attractive potential field is given by

$$
U_{a}\left(\mathbf{P}_{\text {cur }}\right)=\frac{1}{2} k_{a} d_{a}\left(\mathbf{P}_{\text {cur }}, \mathbf{P}_{\text {tar }}\right)^{2},
$$

where $k_{a}$ is a positive gain and $d_{a}\left(\mathbf{P}_{\text {cur }}, \mathbf{P}_{\text {tar }}\right)$ is the minimum distance between current position and the target position, in a $2 \mathrm{D}$ case defined as

$$
d_{a}=\sqrt{\left(x_{\mathrm{cur}}-x_{\mathrm{tar}}\right)^{2}+\left(y_{\mathrm{cur}}-y_{\mathrm{tar}}\right)^{2}} .
$$


Input: the current position $\mathbf{P}_{\text {cur }}\left(x_{c u r}, y_{\text {cur }}\right)$ of UAV, target position $\mathbf{P}_{\text {tar }}\left(x_{t a r}, y_{t a r}\right)$, obstacles position;

Output: The path to the target;

Calculate $d_{a}$;

Update path matrix $M \longleftarrow \mathbf{P}_{\text {cur; }}$;

while $d_{a}>c$ ( $c$ is a small positive constant) do

Calculate attractive force $F_{a}$, using (2) and (5);

Calculate repulsive force $F_{r i}$, using (4) and (6);

Calculate the components of attractive force along the $x$ and $y$ directions, respectively;

Calculate the components of repulsive force along the $x$ and $y$ directions, respectively;

Calculate the next point on the path $\mathbf{P}_{\text {next }}$ according to the resultant of attractive force and repulsive force; end while Update $M \longleftarrow \mathbf{P}_{\text {next }}$;

Algorithm 1: Path planning algorithm based on TAPF

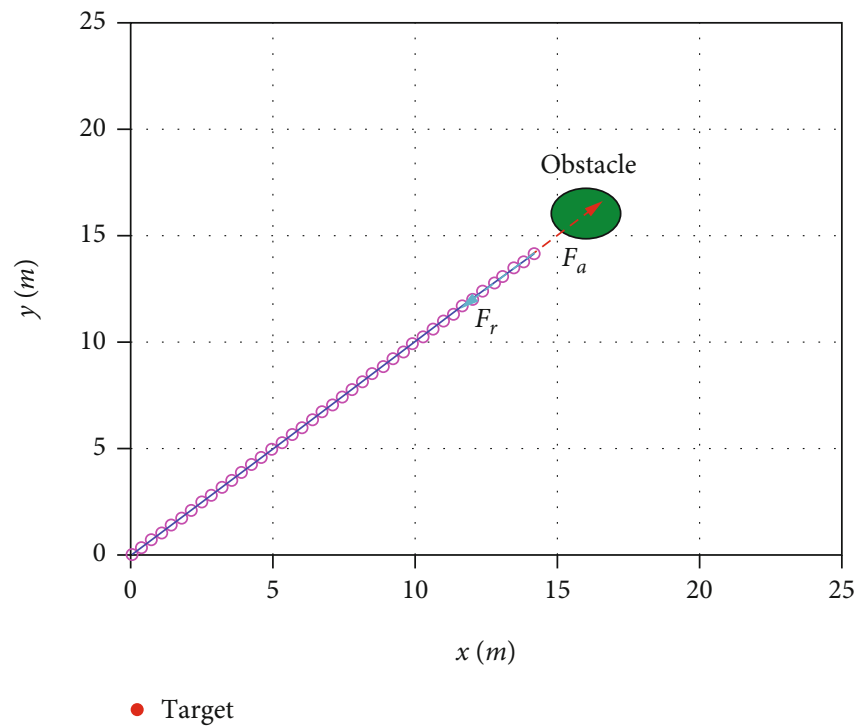

FIgURE 1: Scenario 1: the local minimum of TAPF with a single obstacle.

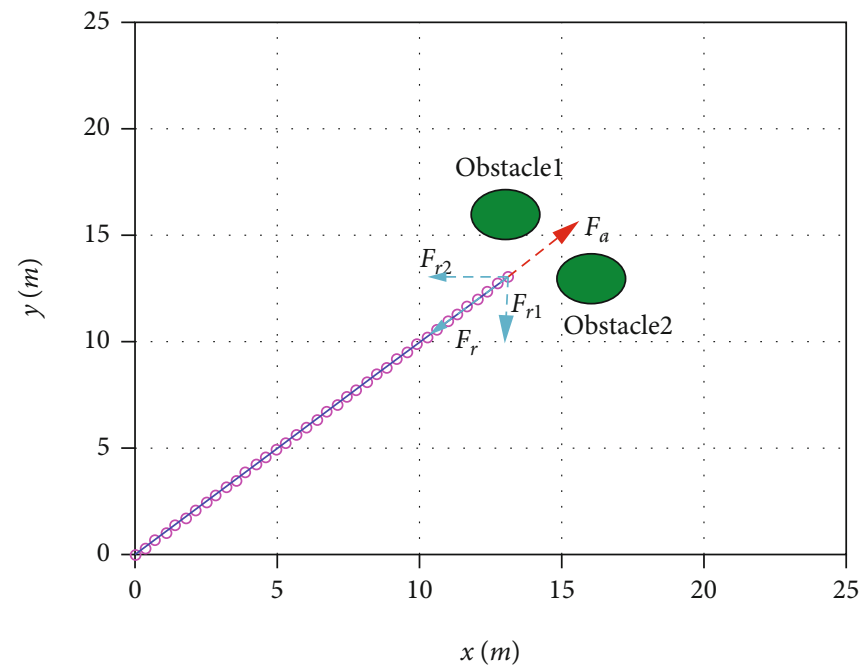

- Target

FIGURE 2: Scenario 1: the local minimum of TAPF with multiple obstacles. 


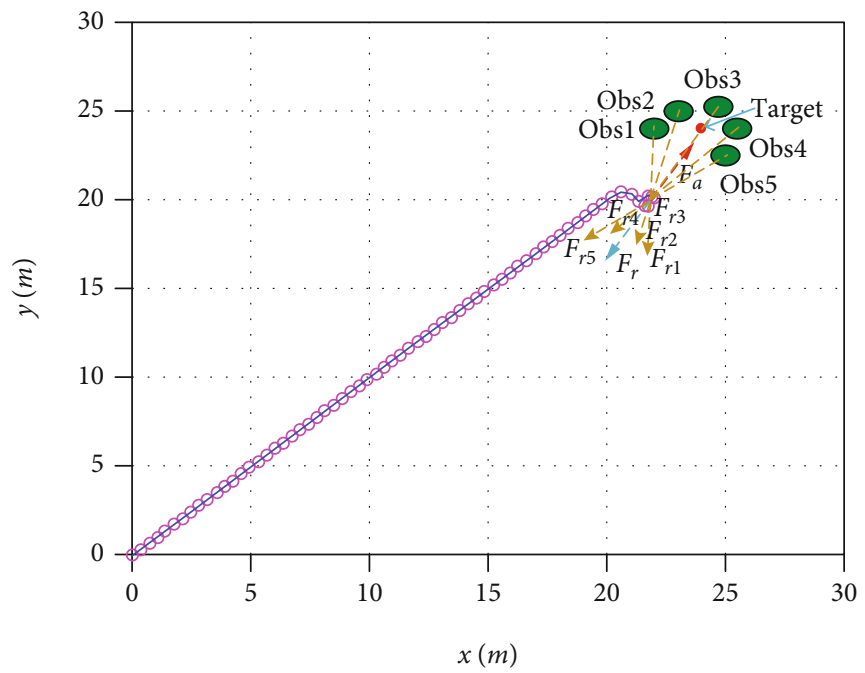

Figure 3: Scenario 1: the unreachable goal of TAPF.

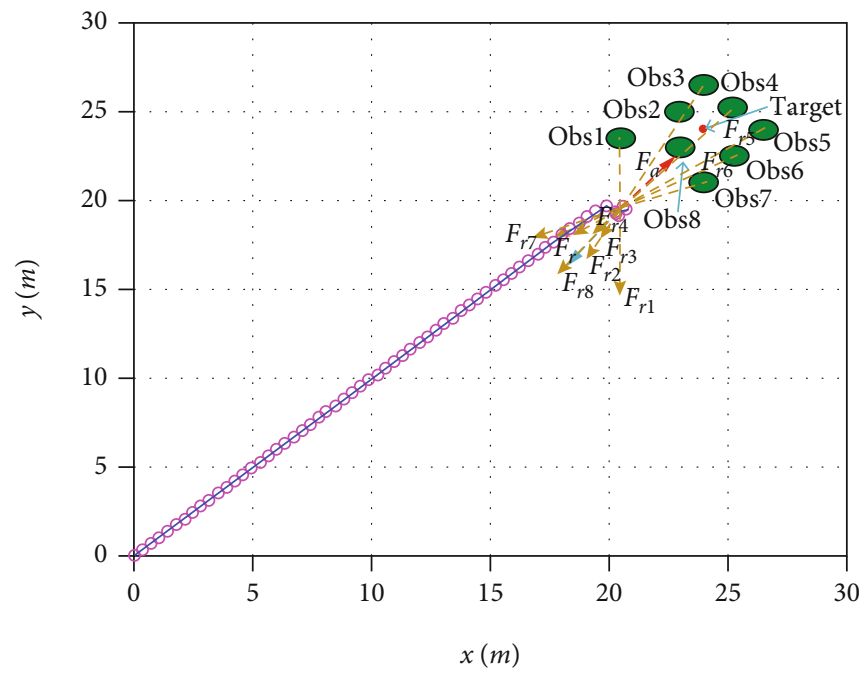

FIGURE 4: Scenario 2: the unreachable goal of TAPF.

Let $\mathbf{P}_{\mathbf{o b} i}\left(x_{\mathrm{ob}_{i}}, y_{\mathrm{ob}_{i}}\right)$ denote position of the ith obstacle, where $i \in N+$. Then, the repulsive potential field of the $i$ th obstacle is defined as

$$
U_{r i}\left(\mathbf{P}_{\mathbf{c u r}}\right)= \begin{cases}\frac{1}{2} k_{r i}\left(\frac{1}{d_{r i}\left(\mathbf{P}_{\mathbf{c u r}}, \mathbf{P}_{\mathbf{o b} i}\right)}-\frac{1}{d_{0}}\right)^{2} & d_{r i} \leq d_{0}, \\ 0 & d_{r i}>d_{0},\end{cases}
$$

where $k_{r i}$ and $d_{0}$ are positive constants and $d_{r i}\left(\mathbf{P}_{\mathbf{c u r}}, \mathbf{P}_{\mathbf{o b} i}\right)$ is the minimum distance between current position and the $i$ th obstacle, in a $2 \mathrm{D}$ case defined as

$$
d_{r i}=\sqrt{\left(x_{c u r}-x_{\mathrm{ob}_{i}}\right)^{2}+\left(y_{c u r}-y_{\mathrm{ob}_{i}}\right)^{2}} .
$$

It is worth noting that $d_{0}$ in (3) shows the influence range of the repulsive potential field of obstacle. Obviously, the attractive potential field is global, while the repulsive potential field is local. Furthermore, the attractive force from the target is obtained by (1)

$$
F_{a}\left(\mathbf{P}_{\text {cur }}\right)=k_{a} d_{a}\left(\mathbf{P}_{\text {cur }}, \mathbf{P}_{\text {tar }}\right)
$$

Meanwhile, the repulsive force of the ith obstacle is obtained from (3):

$$
F_{r i}\left(\mathbf{P}_{\mathrm{cur}}\right)= \begin{cases}k_{r i}\left(\frac{1}{d_{r i}}-\frac{1}{d_{0}}\right)\left(\frac{1}{d_{r i}}\right)^{2} & d_{r i} \leq d_{0} \\ 0 & d_{r i}>d_{0} .\end{cases}
$$

Therefore, the path planning for UAV based on the TAPF algorithm is shown in Algorithm 1. 


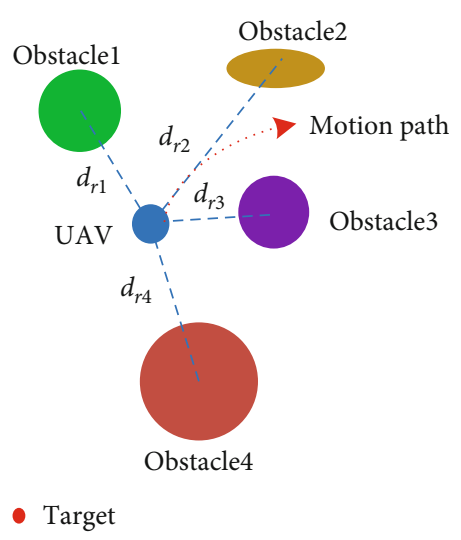

FigURE 5: UAV moves around the nearest obstacle.

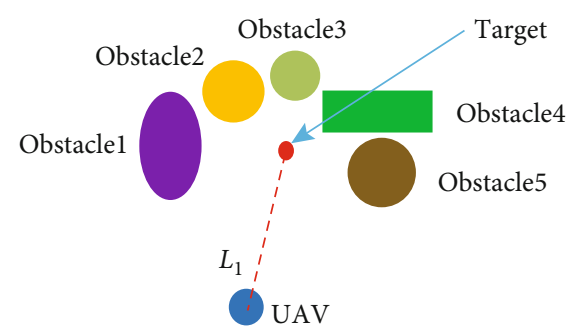

FIGURE 6: Example 1: the parallel search strategy for UAV when the goal is unreachable.

2.2. Local Minimum. The local minimum is an inherent disadvantage of TAPF. When the attractive force and repulsive force reach a balance, the UAV would encounter a trap of a local minimum, which means that the UAV stops moving towards target, as shown in Figures 1 and 2, where $F_{a}$ and $F_{r}$ represent the attractive force and resultant repulsive force at the current position, respectively, and $F_{r i}, i=1,2$, represents repulsive force of the $i$ th obstacle. It is evident that a single obstacle or multiple obstacles may cause UAV to fall into a local minimum when a balance of the attractive force and repulsive force is reached.

2.3. Unreachable Target. Another shortcoming of TAPF is that the goal might be inaccessible for UAV, when obstacles are near the target, as shown in Figures 3 and 4, where Obsi $, i=1, \cdots, 8$, denotes the $i$ th obstacle. In fact, the attraction of the goal to UAV is gradually decreasing, as the UAV approaches destination from (5), while the repulsive force of obstacles to UAV is gradually increasing. Therefore, the UAV fails to plan a path to destination using TAPF.

\section{Novel APF Based on Parallel Search}

To address the problems of the local minimum and unreachable goal of TAPF, a novel parallel search-based APF algorithm is proposed to achieve path planning of a UAV. Such an algorithm consists of two key ideas. The first idea is that UAV moves around the nearest obstacle when it encounters a local minimum, as shown in Figure 5, from which it is observed that $d_{r 3}$ is smallest. To avoid collision with other obstacles, the UAV makes a circular motion with radius $d_{r 3}$

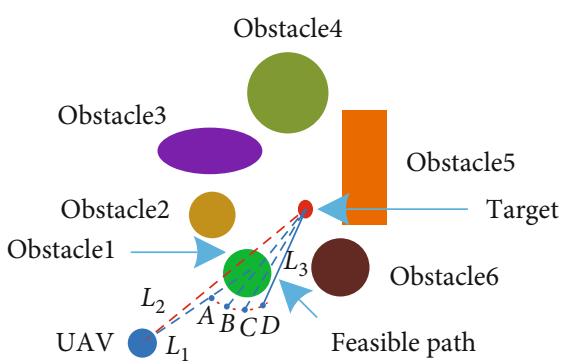

Figure 7: Example 2: the parallel search strategy for UAV when the goal is unreachable.

around the third obstacle. Then, the balance of the attractive force and repulsive force will be broken once the UAV moves a step around the third obstacle, which would guide the UAV to escape from the local minimum under the APF framework.

The second idea of the proposed algorithm is that when the goal is unreachable for the UAV, the movement around the nearest obstacle and the detection of obstacle between the current position and target position will be performed in parallel. Two examples in Figures 6 and 7 are used to further illustrate this idea.

In Figure $6, L_{1}$ represents a line that goes through the current position of UAV and target position. When the target is unreachable for UAV, the points on the line $L_{1}$ will be scanned continuously to find the intersection points of the line $L_{1}$ and the obstacle. If the number of intersection points is equal to zero, it means the UAV can move directly to the target.

In the following, we will explain the scenario in Figure 7, where $L_{2}$ represents a line that goes through the current position of UAV and target position and $\bar{L}$ denotes a line connecting the current position of UAV and the obstacle. It is obvious that the number of intersection points of $L_{2}$ and the obstacle is greater than zero. In these circumstances, the UAV moves firstly along the line $\bar{L}$ to the point $A\left(x_{A}, y_{A}\right)$. Then, the UAV moves to the point $D$ around the first obstacle with radius $R_{A}$, where

$$
R_{A}=\sqrt{\left(y_{o b 1}-y_{A}\right)^{2}+\left(x_{o b 1}-x_{A}\right)^{2}} .
$$

Finally, the UAV successfully reaches the destination along the feasible path $L_{3}$. It is worth noting that the detection of the obstacle between the current position and target position will also be performed simultaneously when UAV is at point $B$ or $C$.

In summary, the flow of the proposed algorithm is shown in Algorithm 2.

Remark 1. In simulation, it is easy to judge the local minimum and the unreachable goal, since the obstacle position and the target position are known. However, the obstacle position may be unknown in real experiments. Therefore, the UAV firstly moves one step around the nearest obstacle within the scanning range of sensor of UAV when it cannot continue moving towards the target. If the current position 


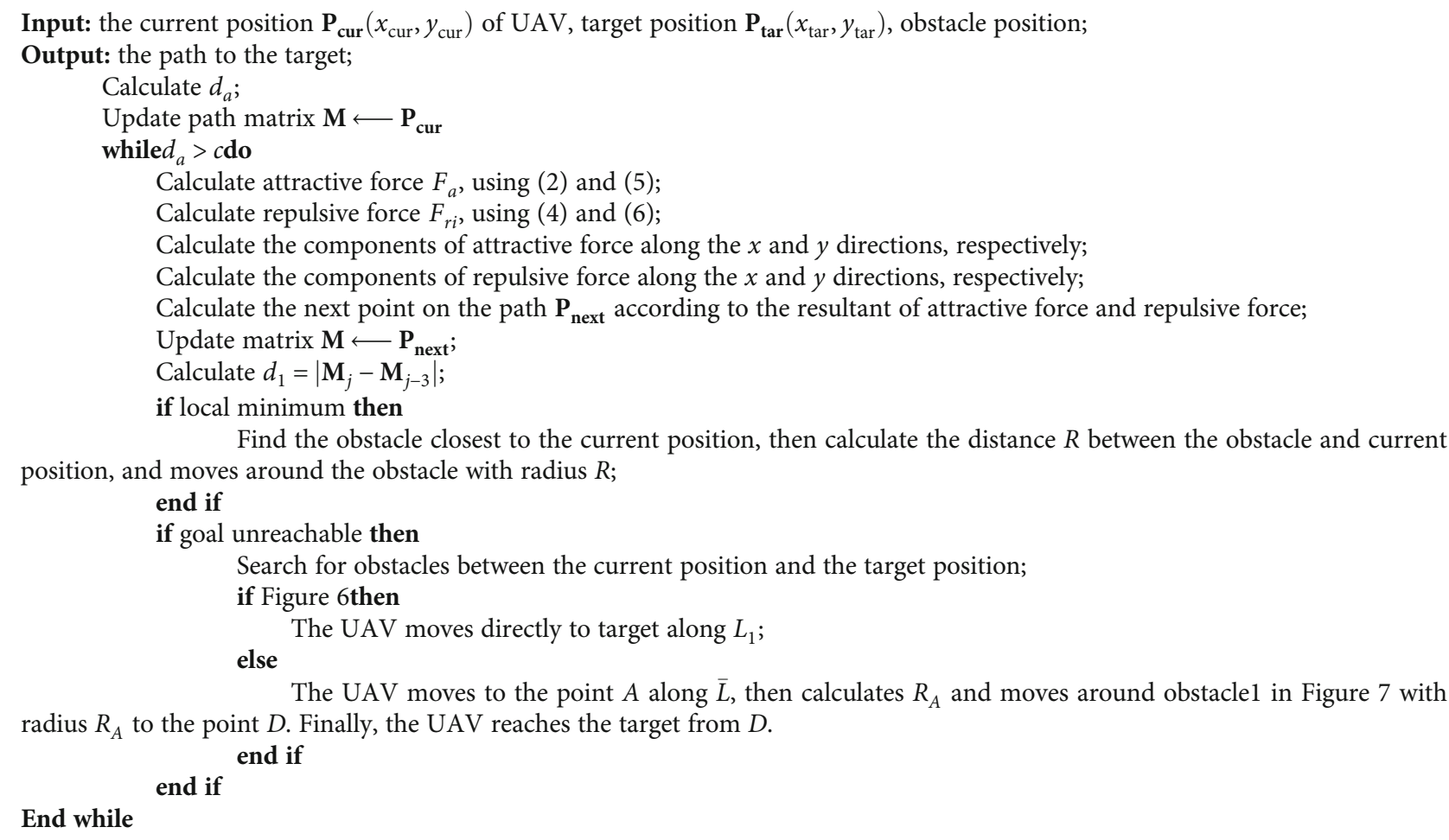

Algorithm 2: A novel APF algorithm based on parallel search for path planning of UAV

is a local minimum, the balance of forces will be broken and the UAV will escape this trap. Otherwise, the UAV moves to an unreachable target.

\section{Controller Design Based on NDO}

4.1. Mathematical Model of Quadrotor UAV. To track the planned path in 2D space, only the attitude angles need to be regulated. Therefore, attitude dynamics of the quadrotor subject to external disturbances are introduced here.

$$
\begin{aligned}
& \ddot{\phi}=\frac{\left(J_{y}-J_{z}\right) \dot{\theta} \dot{\varphi}-J_{r} \dot{\theta} \Omega+L F_{\phi}}{J_{x}}+\bar{d}_{\phi}, \\
& \ddot{\theta}=\frac{\left(J_{z}-J_{x}\right) \dot{\phi} \dot{\varphi}+J_{r} \dot{\phi} \Omega+L F_{\theta}}{J_{y}}+\bar{d}_{\theta}, \\
& \ddot{\varphi}=\frac{\left(J_{x}-J_{y}\right) \dot{\phi} \dot{\theta}+f F_{\varphi}}{J_{z}}+\bar{d}_{\varphi},
\end{aligned}
$$

where $[\phi, \theta, \varphi]$ are altitude, roll angle, pitch angle and yaw angle of quadrotor, respectively; $F_{\chi}, \chi=\phi, \theta, \varphi$ are the control inputs of the system; $L, f, J_{r}, J_{n}, n=x, y, z$ denote the distance from rotor center to mass center, force to moment factor, inertia of each propeller, inertias of the quadrotor around $x$-, $y$ - and $z$-axes, respectively; and $\Omega$ is the difference in angular speed of the rotors on the diagonal of the quadrotor. The terms $\bar{d}_{\phi}, \bar{d}_{\theta}$, and $\bar{d}_{\varphi}$ denote the effect of wind on the translational and rotational subsystems of the quadrotor in the form of external disturbances. Compared with brushless motor, the propeller of quadrotor is very light; therefore, the terms $J_{r} \dot{\theta} \Omega / J_{x}$ and $J_{r} \dot{\phi} \Omega / J_{y}$ are ignored here.

4.2. Assumptions. To make the subsequent analysis rigorous, the following assumption is given.

Assumption 1. It is assumed that the disturbances change slowly, i.e., $\dot{d}_{\phi}=\dot{d}_{\theta}=\dot{d}_{\varphi}=0$.

Assumption 2. In the design of controller for the quadrotor, to avoid any singularity, we set $-\pi / 2<\phi<\pi / 2$ and $-\pi / 2<\theta$ $<\pi / 2$.

4.3. Observer Design. Some of the involved disturbance components in (8) are redefined as $d_{\phi}=J_{x} \bar{d}_{\phi}, d_{\theta}=J_{y} \bar{d}_{\theta}$, and $d_{\varphi}=J_{z} \bar{d}_{\varphi}$. To compensate for external disturbance, a NDO with exponential convergence is proposed. Define observation error as

$$
\tilde{d}_{\chi}=d_{\chi}-\widehat{d}_{\chi}
$$

where $\widehat{d}_{\chi}$ is the estimate of $d_{\chi}$ with $\chi=\phi, \theta, \varphi$. Considering Assumption 1, the derivative of observation error $\tilde{d}_{\chi}$ in (9) in attitude channel is obtained by

$$
\dot{\tilde{d}}_{\chi}=-\dot{\vec{d}}_{\chi}
$$




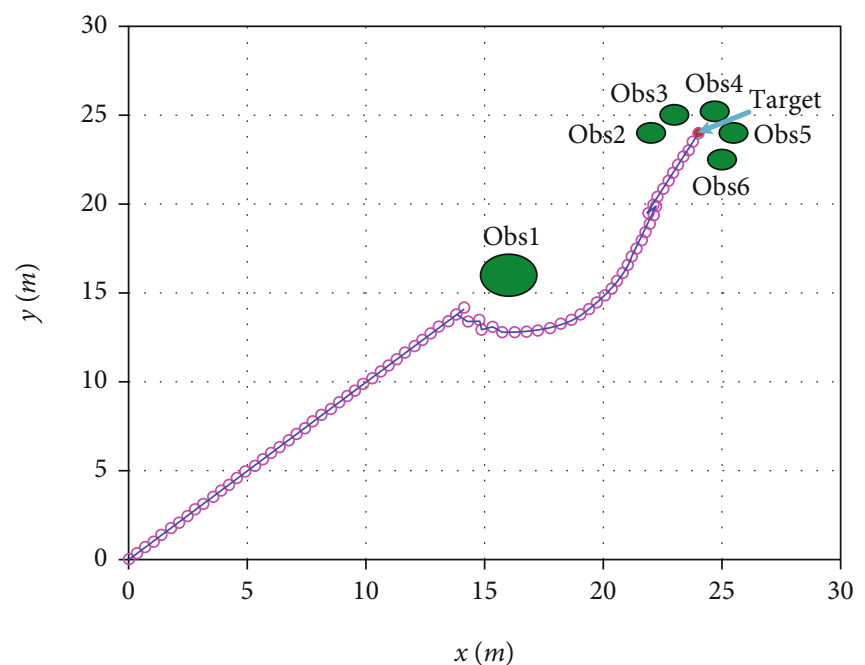

FIGURE 8: Scenario 1: the result of path planning of the proposed algorithm.

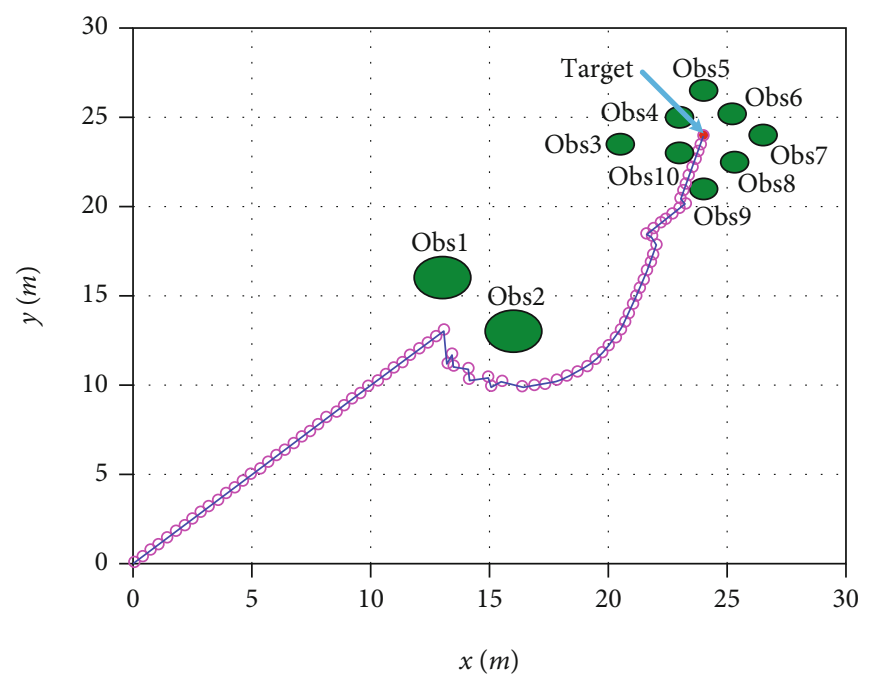

FIGURE 9: Scenario 2: the result of path planning of the proposed algorithm.

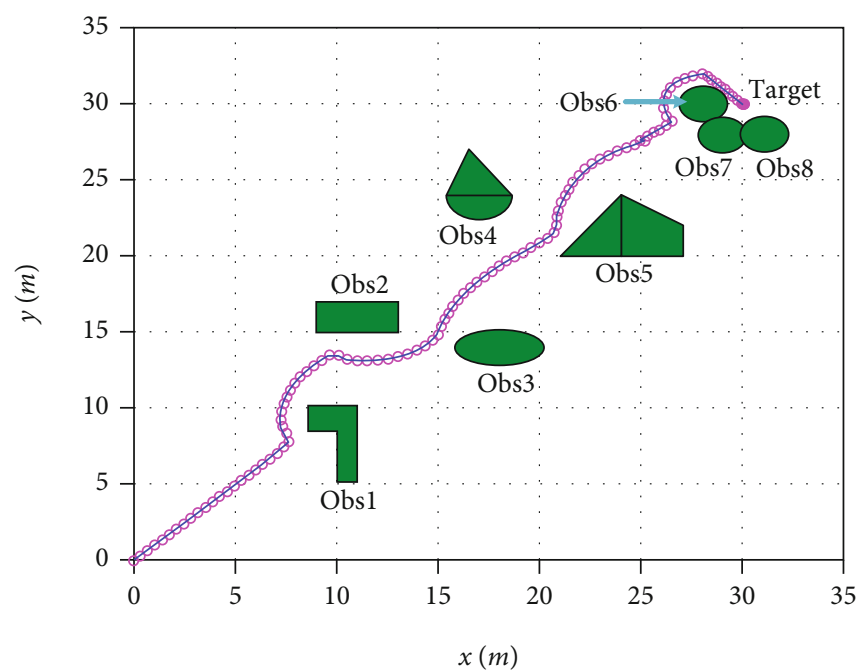

FIGURE 10: Scenario 3: the result of path planning of the proposed algorithm in a complex environment. 
TABLE 1: The simulation parameters in Figures 8-10.

\begin{tabular}{lccc}
\hline Parameter & Figure 8 & Figure 9 & Figure 10 \\
\hline Start position & $(0,0)$ & $(0,0)$ & $(0,0)$ \\
Target position & $(24,24)$ & $(24,24)$ & $(32,32)$ \\
Obstacle1 position & $(16,16)$ & $(13,16)$ & $(10,9)$ \\
Obstacle2 position & $(22,24)$ & $(16,13)$ & $(11,16)$ \\
Obstacle3 position & $(23,25)$ & $(20.5,23.5)$ & $(18,14)$ \\
Obstacle4 position & $(24.7,25.2)$ & $(23,25)$ & $(17,24)$ \\
Obstacle5 position & $(25.5,24)$ & $(24,26.5)$ & $(24,22)$ \\
Obstacle6 position & $(25,22.5)$ & $(25.2,25.2)$ & $(28,30)$ \\
Obstacle7 position & $\times$ & $(26.5,24)$ & $(29,28)$ \\
Obstacle8 position & $\times$ & $(25.3,22.5)$ & $(31,28)$ \\
Obstacle9 position & $\times$ & $(24,21)$ & $\times$ \\
Obstacle10 position & $\times$ & $(23,23)$ & $\times$
\end{tabular}

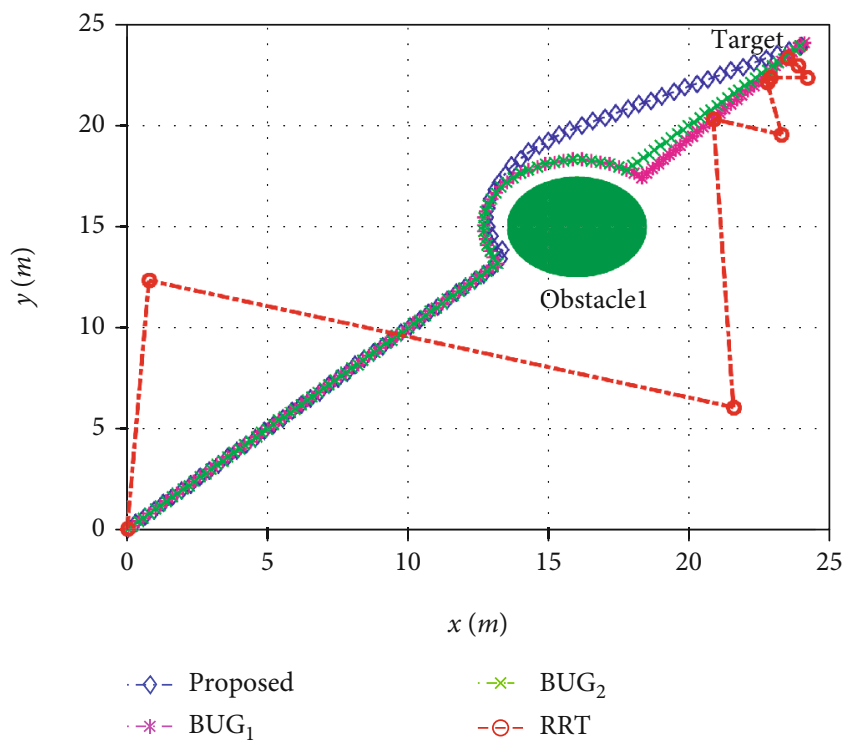

FIgURE 11: Scenario 1: the result of path planning of the four algorithms with an obstacle.

Then, the NDO in attitude channel is designed as

$$
\begin{array}{cc}
\dot{x}_{\chi}= & P_{\chi}\left(W_{j}-Y_{j}\right)-P_{\chi} \widehat{d}_{\chi}, \\
\widehat{d}_{\chi}= & x_{\chi}+P_{\chi} J_{n} \dot{\chi},
\end{array}
$$

where $j=1,2,3, \quad n=x, y, z, \quad W_{1}=\left(J_{z}-J_{y}\right) \dot{\theta} \dot{\varphi}, \quad Y_{1}=L U_{2}$, $W_{2}=\left(J_{x}-J_{z}\right) \dot{\phi} \dot{\varphi}, \quad Y_{2}=L U_{3}, \quad W_{3}=\left(J_{y}-J_{x}\right) \dot{\phi} \dot{\theta}, \quad Y_{3}=f U_{4}$, $x_{\chi}$ is an auxiliary variable, and $P_{\chi}$ is a positive gain.

Theorem 2. If Assumption 1 holds and the observer is designed as (11), then the observation error $\tilde{d}_{z}$ in (9) will exponentially converge to zero.

Proof. See Appendix.

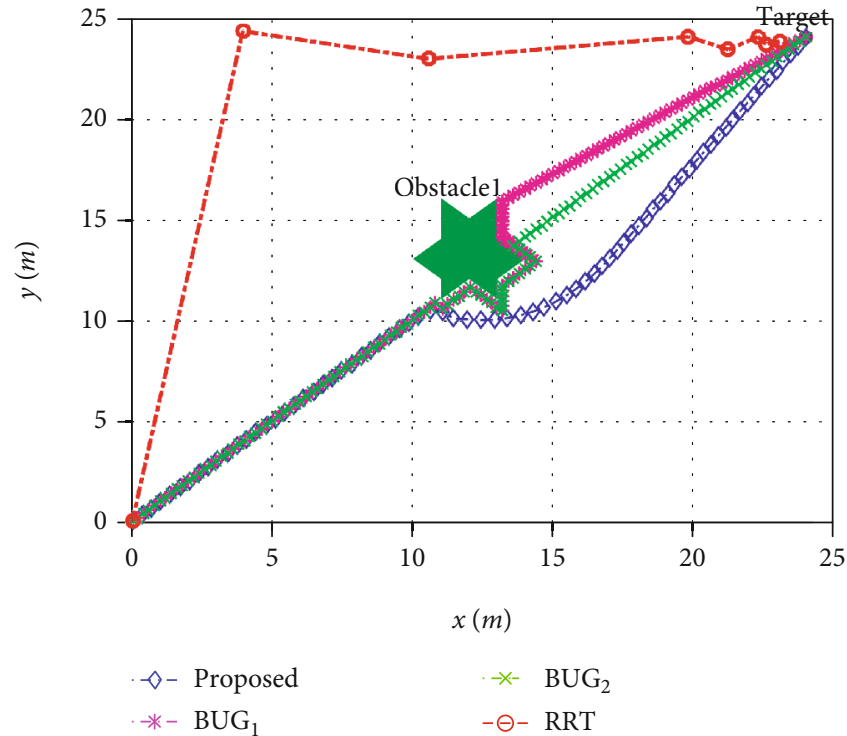

FIgURE 12: Scenario 2: the result of path planning of the four algorithms with an obstacle.

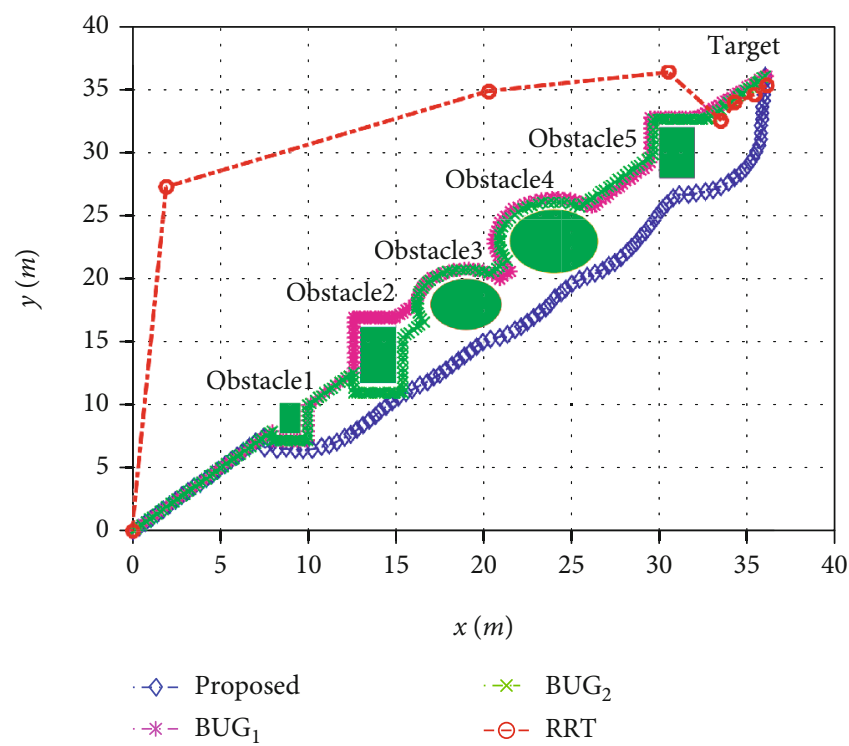

FIgURE 13: Scenario 1: the result of path planning of the four algorithms with multiple obstacles.

Remark 3. Note that $\widehat{d}_{\chi}$ is not the direct estimates of $\bar{d}_{\chi}$ in (8) from (9). If we define $\breve{d}_{\chi}=\widehat{d}_{\chi} / \varepsilon, \varepsilon=J_{x}, J_{y}, J_{z}$, then $\breve{d}_{i}$ denote the estimates of $\bar{d}_{\chi}$ in (8).

4.4. Controller Design. To address the problem of tracking control in the attitude channel, a backstepping scheme is proposed. We define the tracking error of attitude as

$$
e_{\chi}=\chi_{d}-\chi
$$




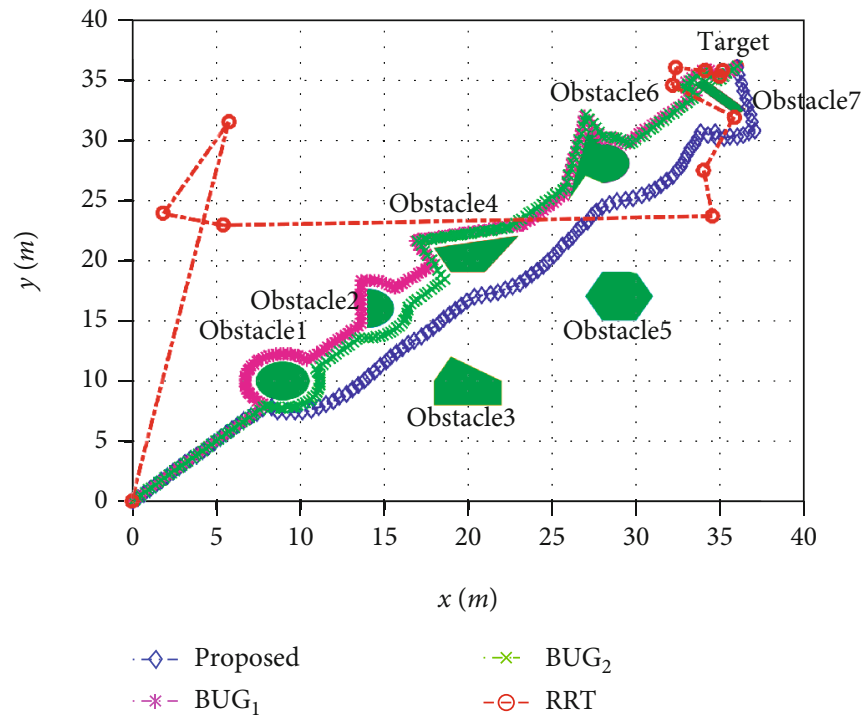

FIGURE 14: Scenario 2: the result of path planning of the four algorithms with multiple obstacles.

TABLE 2: The simulation parameters in Figures 11-14.

\begin{tabular}{lcccc}
\hline Parameter & Figure 11 & Figure 12 & Figure 13 & Figure 14 \\
\hline Start position & $(0,0)$ & $(0,0)$ & $(0,0)$ & $(0,0)$ \\
Target position & $(24,24)$ & $(24,24)$ & $(36,36)$ & $(36,36)$ \\
Obstacle1 position & $(16,15)$ & $(12,13)$ & $(9,9)$ & $(9,10)$ \\
Obstacle2 position & $\times$ & $\times$ & $(14,14)$ & $(14,16)$ \\
Obstacle3 position & $\times$ & $\times$ & $(19,18)$ & $(20,10)$ \\
Obstacle4 position & $\times$ & $\times$ & $(24,23)$ & $(20,20)$ \\
Obstacle5 position & $\times$ & $\times$ & $(31,30)$ & $(29,17)$ \\
Obstacle6 position & $\times$ & $\times$ & $\times$ & $(28,28)$ \\
Obstacle7 position & $\times$ & $\times$ & $\times$ & $(35,34)$ \\
\hline
\end{tabular}

where $\chi_{d}$ is the desired altitude signal. Then, the attitude controller is designed as

$$
F_{\chi}=R_{j}+G_{j}\left(c_{\chi 1} \dot{e}_{\chi}+\ddot{\chi}_{d}+e_{\chi}+c_{\chi 2} e_{\chi 1}\right)+Q_{j},
$$

where $R_{1}=\left(\left(J_{z}-J_{y}\right) / L\right) \dot{\theta} \dot{\varphi}, G_{1}=J_{x} / L, Q_{1}=\widehat{d}_{\phi} / L, R_{2}=\left(\left(J_{x}\right.\right.$ $\left.\left.-J_{z}\right) / L\right) \dot{\phi} \dot{\varphi}, G_{2}=J_{y} / L, Q_{3}=\widehat{d}_{\theta} / L, R_{3}=\left(\left(J_{y}-J_{x}\right) / L\right) \dot{\phi} \dot{\theta}, G_{3}$ $=J_{z} / f, Q_{3}=\widehat{d}_{\varphi} / f, c_{\chi 1}, c_{\chi 2}>\lambda_{\chi} / 2$ with $\lambda_{\chi}$ being a positive constant, and

$$
e_{\chi 1}=-\dot{\chi}+c_{\chi 1} e_{\chi}+\dot{\chi}_{d}
$$

Theorem 4. Under Assumption 1 and Assumption 2, for the altitude dynamics in (8), if the control input $F_{\chi}$ is designed as (13), then the tracking error for desired attitude is guaranteed to converge to zero exponentially, i.e., $e_{\chi} \longrightarrow 0$ as $t \longrightarrow$ $\infty$.

Proof. See Appendix.
TABle 3: Time consumption of the four algorithms in Figures 1114 .

\begin{tabular}{lcccc}
\hline Algorithm & Figure 11 & Figure 12 & Figure 13 & Figure 14 \\
\hline Proposed & $0.0567 \mathrm{~s}$ & $0.0554 \mathrm{~s}$ & $0.0748 \mathrm{~s}$ & $0.0947 \mathrm{~s}$ \\
BUG $_{1}$ & $0.0829 \mathrm{~s}$ & $0.0736 \mathrm{~s}$ & $0.1292 \mathrm{~s}$ & $0.1419 \mathrm{~s}$ \\
BUG $_{2}$ & $0.0701 \mathrm{~s}$ & $0.0714 \mathrm{~s}$ & $0.1210 \mathrm{~s}$ & $0.1260 \mathrm{~s}$ \\
RRT & $0.7150 \mathrm{~s}$ & $0.2022 \mathrm{~s}$ & $1.1637 \mathrm{~s}$ & $0.5662 \mathrm{~S}$ \\
\hline
\end{tabular}

TABLe 4: Path length of the four algorithms in Figures 11-14.

\begin{tabular}{lcccc}
\hline Algorithm & Figure 11 & Figure 12 & Figure 13 & Figure 14 \\
\hline Proposed & $35.8800 \mathrm{~m}$ & $35.5200 \mathrm{~m}$ & $56.1371 \mathrm{~m}$ & $56.6288 \mathrm{~m}$ \\
BUG $_{1}$ & $37.1662 \mathrm{~m}$ & $38.5924 \mathrm{~m}$ & $62.7243 \mathrm{~m}$ & $65.8486 \mathrm{~m}$ \\
BUG $_{2}$ & $36.3676 \mathrm{~m}$ & $37.3622 \mathrm{~m}$ & $64.2180 \mathrm{~m}$ & $63.2446 \mathrm{~m}$ \\
RRT & $56.2966 \mathrm{~m}$ & $44.4007 \mathrm{~m}$ & $66.2756 \mathrm{~m}$ & $91.0735 \mathrm{~m}$ \\
\hline
\end{tabular}

\section{Simulation}

5.1. Comparison with TAPF. The simulation parameters including the start position, target position, and obstacle positions in Figures 8-10 are listed in Table 1. To demonstrate the effectiveness of the proposed path planning framework to deal with the traps of local minimum and unreachable goal of TAPF in Figures 1-4, simulations are carried out, as shown in Figures 8 and 9, where we can observe that the UAV can plan a feasible path to the destination with obstacle avoidance. Furthermore, the proposed algorithm is verified in complex environments with multiple obstacles in Figure 10.

5.2. Comparison with $B U G_{1}, B U G_{2}$, and $R R T$. Considering the characteristics of the proposed algorithm, $B G_{1}$, 

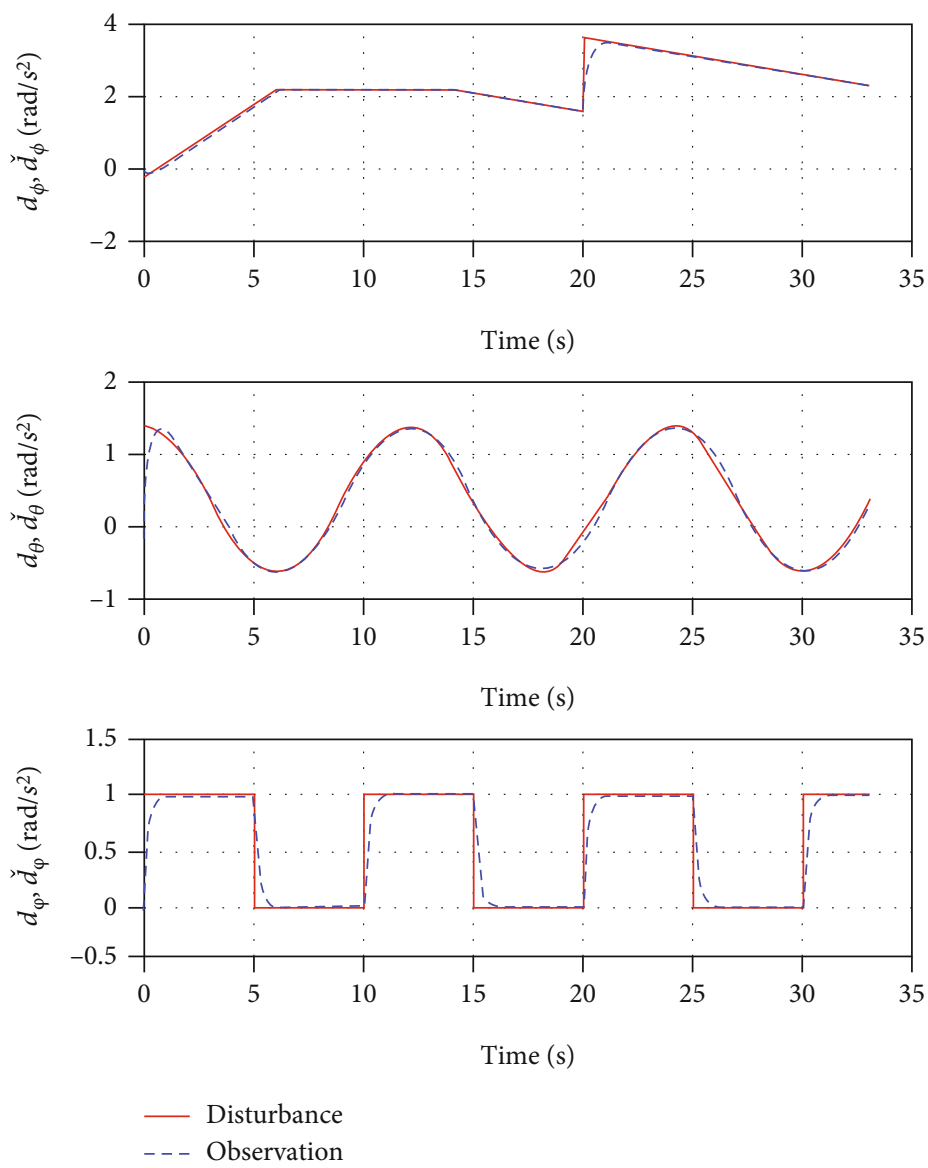

Figure 15: Estimations of external disturbances.

$\mathrm{BUG}_{2}$, and RRT, and to fairly compare their abilities of path planning, the obstacles of different shapes are placed around the line connecting the start point and the target point.

The simulation parameters including the start position, target position, and obstacle positions in Figures 11-14 are shown in Table 2. Furthermore, the comparisons with the three path planning algorithms, namely, $\mathrm{BUG}_{1}, \mathrm{BUG}_{2}$, and RRT, are presented in Figures 11-14. Figures 11 and 12 show the results of path planning of UAV with a single obstacle, while the results of path planning of UAV with multiple obstacles are shown in Figures 13 and 14. From the results, the feasible path to the target with obstacle avoidance can be obtained when the proposed algorithm, $\mathrm{BUG}_{1}, \mathrm{BUG}_{2}$, and RRT are applied to path planning of the UAV, respectively. Table 3 shows the time consumption of the four algorithms. It is obvious that in either the environment with a single obstacle or with multiple obstacles, the time consumption of the proposed algorithm is the least. The path length in Figures 11-14 are listed in Table 4, from which we find that compared with paths generated by $\mathrm{BUG}_{1}, \mathrm{BUG}_{2}$, and RRT, a shorter path is obtained using the proposed algorithm. For the environment with a single obstacle, the shape of obstacle has a greater effect on the length of path provided by $\mathrm{BUG}_{1}$ and $\mathrm{BUG}_{2}$. Meanwhile, RRT has the worst performance in terms of time consumption and path length. Also, the UAV cannot reach the target accurately when RRT is applied to the UAV.

Overall, compared with $\mathrm{BUG}_{1}, \mathrm{BUG}_{2}$, and RRT, the proposed algorithm has advantages in time consumption and path length, which means that less time and energy are required to reach the target for the UAV.

5.3. Trajectory Tracking. The physical parameters of quadrotor are set as follows: $L=0.4 \mathrm{~m}, J_{x}=0.16 \mathrm{kgm}^{2}, J_{y}=0.16$ $\mathrm{kgm}^{2}, J_{z}=0.32 \mathrm{kgm}^{2}$, and $f=0.05 \mathrm{~m}$. The initial attitude of the quadrotor is set as 0rad. Furthermore, to follow the planned path, the desired attitude angles need to be addressed. Figure 11 is used as an example here, whose desired attitude states are calculated as follows: $d_{\phi}=0, d_{\theta 1}=$ $1, d_{\theta 2}=0, d_{\varphi 1}=0.785, d_{\varphi 2}=1.415, d_{\varphi 3}=2.597, d_{\varphi 4}=1.537$, $d_{\varphi 5}=1.865, d_{\varphi 6}=1.617, d_{\varphi 7}=1.514, d_{\varphi 8}=1.399, d_{\varphi 9}=$ $1.288, d_{\varphi 10}=1.181, d_{\varphi 11}=1.011, d_{\varphi 12}=0.985, d_{\varphi 13}=0.813$, $d_{\varphi 14}=0.78, d_{\varphi 15}=0.737, d_{\varphi 16}=0.669, d_{\varphi 17}=0.607, d_{\varphi 18}=$ $0.552, d_{\varphi 19}=0.506$, and $d_{\varphi 20}=0.47$. It should be noted that (1) the yaw angle is used to adjust the forward direction of the quadrotor, and the pitch angle is used to control the forward speed of the quadrotor, while the roll angle is required to maintain at 0 rad and (2) when the yaw angle is trying to maintain one of the above states, a desired pitch 

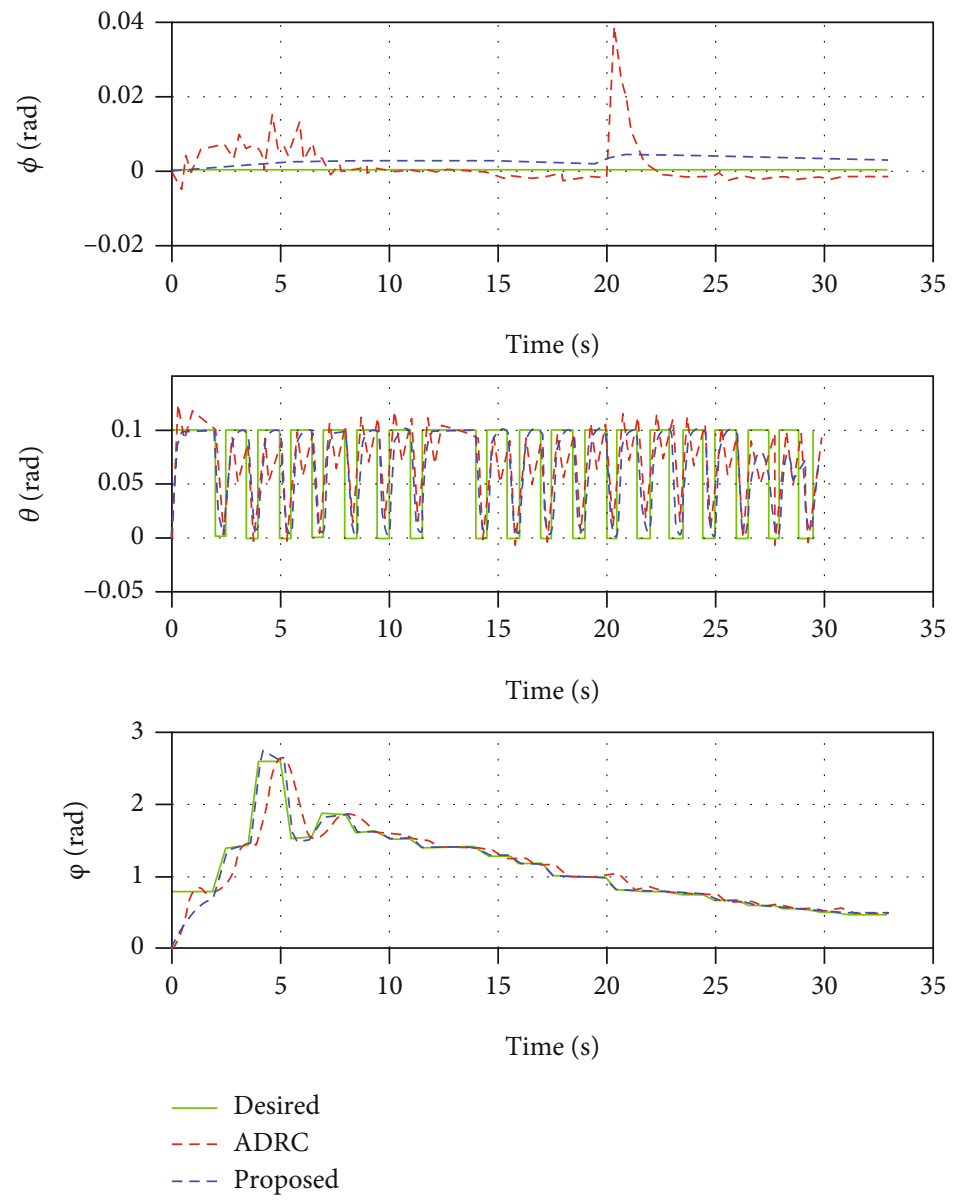

FIGURE 16: Tracking results for reference inputs.

angle of $0.1 \mathrm{rad}$ will be tracked and when the yaw angle is switched between the two states, the desired pitch angle is set as 0rad. In addition, the disturbances in attitude channels are given as

$$
\begin{aligned}
& d_{\phi}= \begin{cases}0.4 t-0.2, & 0 \leq t<6, \\
2.2, & 6 \leq t<14, \\
-0.1 t+3.6, & 14 \leq t<20, \\
-0 . t+5.6, & 20 \leq t<33,\end{cases} \\
& d_{\theta}=\cos \left(\frac{\pi}{6} t\right)+0.4, \\
& d_{\varphi}= \begin{cases}1, & 0 \leq t<5 \text { or } 10 \leq t<15 \cdots, \\
0, & 5 \leq t<10 \text { or } 15 \leq t \leq 20 \cdots .\end{cases}
\end{aligned}
$$

To verify the effectiveness of the proposed NDO, the time-varying disturbance with different frequencies are injected into the quadrotor system. The estimations of external disturbances are shown in Figure 15, where we can see that the disturbances (15), (16), (17) can be estimated, even if the derivatives of the disturbances are assumed to be 0 in the design of the disturbance observer.
However, the disturbance estimations of the pitch and yaw channels have small fluctuations.

The corresponding tracking results for the desired signals are presented in Figure 16. Obviously, tracking and frequent switching of so many states raise a challenging problem for the quadrotor controller, especially in the pitch and yaw channels. However, compared to ADRC, the proposed controller has better tracking performance. In addition, in the roll channel, oscillation is generated in the initial stage, and a large spike is produced at the 20th second when ADRC is applied to the quadrotor, while a smoother tracking performance is provided by the proposed controller.

\section{Conclusion}

In this paper, a novel APF algorithm based on parallel search is proposed for path planning of a UAV. An algorithm for moving around the nearest obstacle is synthesized into APF to avoid the trap of local minimum. A parallel search algorithm is presented to address the problem of an unreachable target. Furthermore, to achieve tracking of planned trajectory of quadrotor UAV subject to external disturbance, a backstepping control strategy with a NDO is designed. The comparative simulations 
are performed to verify the effectiveness of the proposed path planning algorithm and the proposed controller.

\section{Appendix}

\section{A. Proof of Theorem 2}

Proof. From the second equation in (11), one has

$$
\dot{\hat{d}}_{\chi}=\dot{x}_{\chi}+P_{\chi} J_{n} \ddot{\chi}
$$

Substituting the first equation to (A.1) yields

$$
\dot{\hat{d}}_{\chi}=P_{\chi}\left(W_{j}-Y_{j}+J_{n} \ddot{\chi}\right)-P_{\chi} \widehat{d}_{\chi} \text {. }
$$

Considering the relationship $d_{\chi}=J_{n} \bar{d}_{\chi}$ and applying (8)-(17) (A.1), (A.2), we have

$$
\dot{\hat{d}}_{\chi}=P_{\chi}\left(d_{\chi}-\hat{d}_{\chi}\right)=P_{\chi} \tilde{d}_{\chi}
$$

Combining (10), the dynamic equation of observation error is obtained:

$$
\dot{\tilde{d}}_{\chi}+P_{\chi} \tilde{d}_{\chi}=0
$$

Solving (A.4), we get

$$
\tilde{d}_{\chi}(t)=\tilde{d}_{\chi}\left(t_{0}\right) e^{-P_{\chi} t}
$$

where $\tilde{d}_{\chi}\left(t_{0}\right)$ is the initial value of the observation error. (A.5) indicates that the observation error $\tilde{d}_{\chi}$ will exponentially converge to zero as $t \longrightarrow \infty$, i.e., $\widehat{d}_{\chi}$ will exponentially converge to $d_{\chi}$ as $t \longrightarrow \infty$ under Assumption 1 .

This completes the proof.

\section{B. Proof of Theorem 4}

Proof. The whole proof is divided into two steps.

Step 1. We define a Lyapunov function candidate

$$
V_{\chi 1}=\frac{1}{2} J_{n} e_{\chi}^{2}
$$

The time derivative of $V_{\chi 1}$ is

$$
\dot{V}_{\chi 1}=J_{n} e_{\chi} \dot{e}_{\chi}
$$

Substituting (12) and (14) to (17), (A.1)-(B.2), it is obtained that

$\dot{V}_{\chi 1}=J_{n} e_{\chi}\left(\dot{\chi}_{d}-\dot{\chi}\right)=J_{n} e_{\chi}\left(e_{\chi 1}+\dot{\chi}-c_{\chi 1} e_{\chi}-\dot{\chi}\right)=-J_{n} c_{\chi 1} e_{\chi}^{2}+J_{n} e_{\chi} e_{\chi 1}$.

Step 2. We define the following Lyapunov function candidate

$$
V_{\chi}=V_{\chi 1}+\frac{1}{2} J_{n} e_{\chi 1}^{2}
$$

The time derivative of $V_{\chi}$ is

$$
\dot{V}_{\chi}=\dot{V}_{\chi 1}+J_{n} e_{\chi 1} \dot{e}_{\chi 1}
$$

Combining (14) and (B.3), (B.5) can be rewritten as

$\dot{V}_{\chi}=-J_{n} c_{\chi 1} e_{\chi}^{2}+J_{n} e_{\chi} e_{\chi 1}+J_{n} e_{\chi 1}\left(-\ddot{\chi}+c_{\chi 1} \dot{e}_{\chi}+\ddot{\chi}_{d}\right)$.

Substituting (8) into (B.6), we get

$\dot{V}_{\chi}=-J_{n} c_{\chi 1} e_{\chi}^{2}+J_{n} e_{\chi} e_{\chi 1}+J_{n} e_{\chi 1}\left(\frac{R_{j}}{G_{j}}-\frac{F_{\chi}}{G_{j}}+\bar{d}_{\chi}+c_{\chi 1} \dot{e}_{\chi}+\ddot{\chi}_{d}\right)$

Substituting the attitude controller (13) into (B.8) yields

$$
\begin{aligned}
\dot{V}_{z} & =-J_{n} c_{\chi 1} e_{\chi}^{2}+J_{n} e_{\chi} e_{\chi 1}+J_{n} e_{\chi 1}\left(-e_{\chi}-c_{\chi 2} e_{\chi 1}+\frac{1}{J_{n}}\left(d_{\chi}-\widehat{d}_{\chi}\right)\right) \\
& =-J_{n} c_{\chi 1} e_{\chi}^{2}-J_{n} c_{\chi 2} e_{\chi 1}^{2}+e_{\chi 1}\left(d_{\chi}-\widehat{d}_{\chi}\right) .
\end{aligned}
$$

The term $d_{\chi}-\widehat{d}_{\chi}$ will vanish from Theorem 2 as $t \longrightarrow \infty$ . Hence, according to (B.1), (B.4) and the relationship $c_{\chi 1}$, $c_{\chi 2} \geq \lambda_{\chi} / 2$, (B.8) is further derived as

$$
\begin{aligned}
\dot{V}_{\chi} & =-J_{n} c_{\chi 1} e_{\chi}^{2}-J_{n} c_{\chi 2} e_{\chi 1}^{2} \\
& =-\lambda_{\chi} V_{\chi}-\left(c_{\chi 1}-\frac{\lambda_{\chi}}{2}\right) J_{n} e_{\chi}^{2}-\left(c_{\chi 2}-\frac{\lambda_{\chi}}{2}\right) J_{n} e_{\chi 1}^{2} \leq-\lambda_{\chi} V_{\chi} .
\end{aligned}
$$

Solving (B.9), it is obtained

$$
V_{\chi}(t) \leq V_{\chi}(0) e^{-\lambda_{\chi} t}
$$

where $V_{\chi}(0)$ is the initial value of $V_{\chi}(t)$. From (B.10), it is concluded that $e_{\chi}$ will exponentially converge to zero as $t$ $\longrightarrow \infty$.

This completes the proof.

\section{Data Availability}

The data used to support this study are included within the article.

\section{Conflicts of Interest}

The authors declare that they have no conflicts of interest. 


\section{Acknowledgments}

The work was supported by the National Natural Science Foundation of China under Grants 61773323, U1934221, and 61733015 .

\section{References}

[1] F. Outay, H. A. Mengash, and M. Adnan, "Applications of unmanned aerial vehicle (UAV) in road safety, traffic and highway infrastructure management: recent advances and challenges," Transportation Research Part A, vol. 141, pp. 116-129, 2020.

[2] B. D. Song, K. Park, and J. Kim, "Persistent uav delivery logistics: Milp formulation and efficient heuristic," Computers and Industrial Engineering, vol. 120, pp. 418-428, 2018.

[3] A. Navarro, M. Young, B. Allan, P. Carnell, P. Macreadie, and D. Ierodiaconou, "The application of unmanned aerial vehicles (UAVs) to estimate above-ground biomass of mangrove ecosystems," Remote Sensing of Environment, vol. 242, article 111747, 2020.

[4] G. M. Hoffmann, H. Huang, S. L. Waslander, and C. J. Tomlin, "Precision flight control for a multi-vehicle quadrotor helicopter testbed," Control Engineering Practice, vol. 19, no. 9, pp. 1023-1036, 2011.

[5] T. Huang, D. Huang, and D. Luo, "Attitude tracking for a quadrotor UAV based on fuzzy PID controller," in 5th International Conference on Information, Cybernetics, and Computational Social Systems, pp. 1-6, Zhengjiang, China, 2018.

[6] K. Chang, Y. Xia, K. Huang, and D. Ma, "Obstacle avoidance and active disturbance rejection control for a quadrotor," $\mathrm{Neu}$ rocomputing, vol. 190, pp. 60-69, 2016.

[7] Y. Zhang, Z. Chen, X. Zhang, Q. Sun, and M. Sun, “A novel control scheme for quadrotor UAV based upon active disturbance rejection control," Aerospace Science and Technology, vol. 79, pp. 601-609, 2018.

[8] T. Huang, D. Huang, Z. Wang, X. Dai, and A. Shah, “Generic adaptive sliding mode control for a quadrotor UAV system subject to severe parametric uncertainties and fully unknown external disturbance," International Journal of Control, Automation and Systems, vol. 19, no. 2, pp. 698-711, 2021.

[9] H. Ríos, R. Falcón, O. A. González, and A. Dzul, "Continuous sliding-mode control strategies for quadrotor robust tracking: real-time application," IEEE Transactions on Industrial Electronics, vol. 66, no. 2, pp. 1264-1272, 2019.

[10] B. Tian, J. Cui, H. Lu, Z. Zuo, and Q. Zong, "Adaptive finitetime attitude tracking of quadrotors with experiments and comparisons," IEEE Transactions on Industrial Electronics, vol. 66, no. 12, pp. 9428-9438, 2019.

[11] X. Lyu, J. Zhou, H. Gu, Z. Li, S. Shen, and F. Zhang, "Disturbance observer based hovering control of quadrotor tailsitter VTOL UAVs using $H_{\infty}$ synthesis," IEEE Robotics and Automation Letters, vol. 3, no. 4, pp. 2910-2917, 2018.

[12] A. Poultney, C. Kennedy, G. Clayton, and H. Ashrafiuon, "Robust tracking control of quadrotors based on differential flatness: simulations and experiments," IEEE/ASME Transactions on Mechatronics, vol. 23, no. 3, pp. 1126-1137, 2018.

[13] N. Koksal, M. Jalalmaab, and B. Fidan, "Adaptive linear quadratic attitude tracking control of a quadrotor UAV based on IMU sensor data fusion," Sensors, vol. 19, no. 1, pp. 46-123, 2019.
[14] F. Chen, W. Lei, K. Zhang, G. Tao, and B. Jiang, "A novel nonlinear resilient control for a quadrotor UAV via backstepping control and nonlinear disturbance observer," Nonlinear Dynamics, vol. 85, no. 2, pp. 1281-1295, 2016.

[15] C. Xu, H. Duan, and F. Liu, "Chaotic artificial bee colony approach to uninhabited combat air vehicle (UCAV) path planning," Aerospace Science and Technology, vol. 14, no. 8, pp. 535-541, 2010.

[16] C. Lin, G. Han, X. Qi, J. du, T. Xu, and M. Martinez-Garcia, "Energy-optimal data collection for unmanned aerial vehicleaided industrial wireless sensor network-based agricultural monitoring system: a clustering compressed sampling approach," IEEE Transactions on Industrial Informatics, vol. 17, no. 6, pp. 4411-4420, 2021.

[17] X. Wu, W. Bai, Y. Xie, X. Sun, C. Deng, and H. Cui, “A hybrid algorithm of particle swarm optimization, metropolis criterion and RTS smoother for path planning of UAVs," Applied Soft Computing, vol. 73, pp. 735-747, 2018.

[18] N. Bolourian and A. Hammad, "Lidar-equipped UAV path planning considering potential locations of defects for bridge inspection," Automation in Construction, vol. 117, article 103250, 2020.

[19] V. Roberge, M. Tarbouchi, and G. Labonté, "Comparison of parallel genetic algorithm and particle swarm optimization for real-time UAV path planning," IEEE Transactions on Industrial Informatics, vol. 9, no. 1, pp. 132-141, 2013.

[20] C. Xu, M. Xu, and C. Yin, "Optimized multi-UAV cooperative path planning under the complex confrontation environment," Computer Communications, vol. 162, pp. 196-203, 2020.

[21] C. Qu, W. Gai, J. Zhang, and M. Zhong, “A novel hybrid grey wolf optimizer algorithm for unmanned aerial vehicle (UAV) path planning," Knowledge-Based Systems, vol. 194, article 105530, 2020.

[22] K. Li, F. Ge, Y. Han, Y. Wang, and W. Xu, "Path planning of multiple UAVs with online changing tasks by an ORPFOA algorithm," Engineering Applications of Artificial Intelligence, vol. 94, article 103807, 2020.

[23] C. Chen, D. Pi, and Y. Xu, "Neighborhood global learning based flower pollination algorithm and its application to unmanned aerial vehicle path planning," Expert Systems with Applications, vol. 170, article 114505, 2021.

[24] P. Yang, K. Tang, J. A. Lozano, and X. Cao, "Path planning for single unmanned aerial vehicle by separately evolving waypoints," IEEE Transactions on Robotics, vol. 31, no. 5, pp. 1130-1146, 2015.

[25] X. Yu, C. Li, and J. Zhou, "A constrained differential evolution algorithm to solve UAV path planning in disaster scenarios," Knowledge-Based Systems, vol. 204, article 106209, 2020.

[26] J. Park and H. J. Kim, “Online trajectory planning for multiple quadrotors in dynamic environments using relative safe flight corridor," IEEE Robotics and Automation Letters, vol. 6, no. 2, pp. 659-666, 2021.

[27] S. H. Kim, G. E. G. Padilla, K. J. Kim, and K. H. Yu, "Flight path planning for a solar powered uav in wind fields using direct collocation," IEEE Transactions on Aerospace and Electronic Systems, vol. 56, no. 2, pp. 1094-1105, 2020.

[28] C. Yin, Z. Xiao, X. Cao, X. Xi, P. Yang, and D. Wu, “Offline and online search: UAV multi-objective path planning under dynamic urban environment," IEEE Internet of Things Journal, vol. 5, no. 2, pp. 546-558, 2018. 
[29] Y. Liu, Q. Wang, H. Hu, and Y. He, “A novel real-time moving target tracking and path planning system for a quadrotor UAV in unknown unstructured outdoor scenes," IEEE Transactions on Systems Man and Cybernetics Systems, vol. 49, no. 11, pp. 2362-2372, 2019.

[30] C. Liu, S. Zhang, and A. Akbar, "Ground feature oriented path planning for unmanned aerial vehicle mapping," IEEE Journal of Selected Topics in Applied Earth Observations and Remote Sensing, vol. 12, no. 4, pp. 1175-1187, 2019.

[31] H. Wang, J. Wang, G. Ding, J. Chen, F. Gao, and Z. Han, "Completion time minimization with path planning for fixed-wing UAV communications," IEEE Transactions on Wireless Communications, vol. 18, no. 7, pp. 3485-3499, 2019.

[32] Y. Cai, Q. Xi, X. Xing, H. Gui, and Q. Liu, "Path planning for UAV tracking target based on improved a-star algorithm," in International Conference on Industrial Artificial Intelligence, pp. 1-6, Shenyang, China, 2019.

[33] X. Chen, G. Y. Li, and X. M. Chen, "Path planning and cooperative control for multiple UAVs based on consistency theory and Voronoi diagram," in 2017 29th Chinese Control And Decision Conference (CCDC), pp. 881-886, Chongqing, China, 2017.

[34] M. Li, Q. Sun, and M. Zhu, "UAV 3-dimension flight path planning based on improved rapidly-exploring random tree," in 2019 Chinese Control And Decision Conference (CCDC), pp. 921-925, Nanchang, China, 2019.

[35] B. Kovács, G. Szayer, F. Tajti, M. Burdelis, and P. Korondi, “A novel potential field method for path planning of mobile robots by adapting animal motion attributes," Robotics and Autonomous Systems, vol. 82, pp. 24-34, 2016.

[36] U. Orozco-Rosas, O. Montiel, and R. Sepúlveda, "Mobile robot path planning using membrane evolutionary artificial potential field," Applied Soft Computing, vol. 77, pp. 236-251, 2019.

[37] Z. Huang, D. Chu, C. Wu, and Y. He, "Path planning and cooperative control for automated vehicle platoon using hybrid automata," IEEE Transactions on Intelligent Transportation Systems, vol. 20, no. 3, pp. 959-974, 2019.

[38] J. Liu, C. Xu, Z. Wu, and Y. F. Chen, "Intelligent rebar layout in RC building frames using artificial potential field," Automation in Construction, vol. 114, article 103172, 2020.

[39] J. J. Kandathil, R. Mathew, and S. S. Hiremath, “Development and analysis of a novel obstacle avoidance strategy for a multirobot system inspired by the bug-1 algorithm," Simulation: Transactions of The Society for Modeling and Simulation International, vol. 96, no. 10, pp. 807-824, 2020.

[40] S. Yang, X. Wang, Y. Wen, J. Wang, and S. Li, "A new intelligent trajectory planning algorithm based on bug2 algorithm:bridge algorithm," in International Conference on Robotics and Biomimetics, pp. 2079-2084, Dali, China, 2019. 